\title{
Study on Data Processing Algorithm of Complex Curve Based on Point Cloud Data
}

\author{
WANG Ke ${ }^{a}$, LU Haiqing ${ }^{b}$, SUN Xingwei ${ }^{c}$ \\ School of mechanical engineering, Shenyang University of Technology, Shenyang 110870, China \\ awk2222@sina.com, 'luhaiqing668@163.com, 'sunxingw@126.com
}

Keywords: point cloud data; least square method; Lagrange multiplier; segment fitting.

\begin{abstract}
When fitting point cloud data, use polynomial curve fitting method is hard to obtain a better fitting effect. While by using segment curve fitting method, there are defects of unsatisfaction with the continuity and differentiability of a function at the place of segments. Therefore, based on the foundation of traditional least square method curve fitting, this paper propose to use Lagrange multiplier to implement point cloud data fairing processing. Through the inspection of practical data, the precision of this algorithm is higher, which meet the requirements of point cloud data's fairing and provide an effective mathematical algorithm for the fairing processing of point cloud data in reverse engineering.
\end{abstract}

\section{Introduction}

Data fitting is an important method for data processing, and least square method is one of the more commonly used data fitting method[1]. When dealing with the point cloud data, if only use one curve function to fit point cloud data, when the rank of polynomial is too low, the fitting precision can not reach scheduled effect. In order to improve the fitting precision and effect, we need to improve the rank of fitted curve, while if the rank is too high, it will result in complicated calculation and difficult operation, even appear Runge phenomenon, and it is also difficult to get a better fitting effect and precision. If adopt segment fitting, the curve after fitting may discontinuous at the place of segments. In order to effectively solve the above problems, this paper puts forward the scheme of point cloud data fairing processing based on Lagrange multiplier.

\section{Establish mathematics model}

In order to use the advanced manufacturing technology and method, we need to transform products into products mathematical model, and further study the advanced products of the same kind, this scientific technology is called "reverse engineering", also known as "back-engineering"[2]. When transform the physical model into CAD model, in order to ensure that the reverse results are as close as possible with the actual device under test, we need to obtain large numbers of discrete points on the surface of the object to be tested, these discrete points generally called point cloud data[3]. As the original data is huge, therefore, the data fitting often can not realized in the whole domain, but by several times piecewise fitting in different data points[4,5].

Definition: in the segment curve fitting, the points interval a single curve fitting needs is called window. As shown in figure 1 . It use a window with $\mathrm{M}$ points to fit $\mathrm{N}$ data points, there are $\mathrm{N}-\mathrm{M}+1$ windows in total.

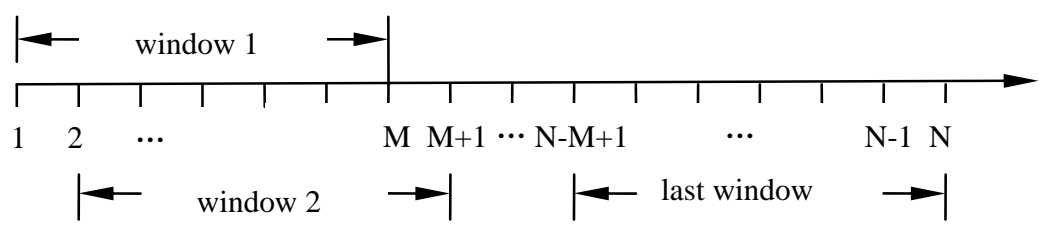

Fig. 1 Segment curve fitting 
The segment curve fitting in this paper is fitted in one window, by output the fitting curve between the first two data, and then the window moving back a data points, made up of the next fitting window and repeat the next fitting process until the last window.

If point cloud data interval is $\left[x_{1}, x_{N}\right]$, set the $\mathrm{N}$ pairs of data point is $\left(x_{j}, y_{j}\right)(j=1,2, \cdots N)$, each window's approximate polynomial is $g(x)$ :

$$
g(x)=\left\{\begin{array}{cc}
g_{1}(x) & x_{1}<x<x_{M} \\
g_{2}(x) & x_{2}<x<x_{M+1} \\
\vdots & \vdots \\
g_{N-M+1}(x) & x_{N-M+1}<x<x_{N}
\end{array} .\right.
$$

In which: in window " 1 " adopt the traditional least square method to fit, then we can get the fitting polynomial $g_{1}(x)$. Suppose the polynomial in window " $\mathrm{k}$ " $(k=2,3,4, \cdots,(N-M+1))$ is:

$$
g_{k}(x)=\sum_{i=0}^{n} a_{k i} x^{i} \quad x_{k}<x<x_{k+M-1} .
$$

During the above formula, $a_{k 0}, a_{k 1}, a_{k 2}, \ldots . ., a_{k n}$ are undetermined coefficient.

To ensure the continuous of curve from $g_{k-1}(x)$ to $g_{k}(x)$ at $x_{k}$ point, the function value at the two places before and after $x_{k}$ are required equal, that is, to add endpoint constraint conditions:

$$
g_{k-1}\left(x_{k}\right)=g_{k}\left(x_{k}\right) \text {. }
$$

At the same time, to ensure the curve's smooth from $g_{k-1}(x)$ to $g_{k}(x)$ at $x_{k}$ point, the first derivative value at the two places before and after $x_{k}$ are also required equal, that is, to add endpoint constraint conditions:

$$
g_{k-1}{ }^{\prime}\left(x_{k}\right)=g_{k}{ }^{\prime}\left(x_{k}\right) \text {. }
$$

\section{The solution of mathematical model}

This mathematical model is a constraint problem with extremum method. This paper by using Lagrange multiplier[6], transform the condition extremum problem into unconditional extreme problems to solve. Take window " $k$ " $(k=2,3,4, \cdots,(N-M+1))$ as an example, the specific methods are as follows:

Introducing Lagrange multiplier $\lambda_{l}(l=1,2)$ and set: $H_{k}=g_{k}\left(x_{k}\right)-g_{k-1}\left(x_{k}\right), \quad K_{k}=g_{k}^{\prime}\left(x_{k}\right)-g_{k-1}^{\prime}\left(x_{k}\right)$, the front modeling result shows that the boundary restriction of the model is:

$$
H_{k}=0, K_{k}=0 \text {. }
$$

Lagrange function is:

$$
Q=\sum_{j=k}^{k+M-1}\left(\sum_{i=0}^{n} a_{k i} x_{j}{ }^{i}-y_{j}\right)^{2}+2 \lambda_{1} H_{k}+2 \lambda_{2} K_{k} \quad x_{k}<x_{j}<x_{k+M-1} .
$$

In order to get the minimal value of $Q$, we can change the size of coefficient $a_{k i}$, thus we need to know the partial derivative of $Q$ to each coefficient, and let their partial derivatives equal zero, namely:

$$
\frac{\partial Q}{\partial a_{k f}}=2 \sum_{j=k}^{k+M-1}\left(\sum_{i=0}^{n} a_{k i} x_{j}{ }^{i}-y_{j}\right) x_{j}{ }^{f}+2 \lambda_{1} \Phi_{f}\left(x_{k}\right)+2 \lambda_{2} \Phi_{f}{ }^{\prime}\left(x_{k}\right) .
$$

In which: $\Phi_{f}(x)=x^{f}, f=(0,1,2,3, \cdots, n)$.

Set: $\frac{\partial Q}{\partial a_{k f}}=0$.

In the form of matrix is: $\left[\begin{array}{cc}S & S_{1} \\ S_{2} & 0\end{array}\right]\left[\begin{array}{l}A \\ \lambda\end{array}\right]=\left[\begin{array}{l}T \\ T_{1}\end{array}\right]$. 
In which: $S=\left[\begin{array}{cccc}\left(\Phi_{0}, \Phi_{0}\right) & \left(\Phi_{0}, \Phi_{1}\right) & \cdots & \left(\Phi_{0}, \Phi_{n}\right) \\ \left(\Phi_{1}, \Phi_{0}\right) & \left(\Phi_{1}, \Phi_{1}\right) & \cdots & \left(\Phi_{1}, \Phi_{n}\right) \\ \vdots & \vdots & & \vdots \\ \left(\Phi_{n}, \Phi_{0}\right) & \left(\Phi_{n}, \Phi_{1}\right) & \cdots & \left(\Phi_{n}, \Phi_{n}\right)\end{array}\right] ; \quad S_{1}=\left[\begin{array}{ccc}\Phi_{0}\left(x_{k}\right) & \Phi_{0}^{\prime}\left(x_{k}\right) \\ \Phi_{1}\left(x_{k}\right) & \Phi_{1}^{\prime}\left(x_{k}\right) \\ \vdots & \vdots \\ \Phi_{n}\left(x_{k}\right) & \Phi_{n}^{\prime}\left(x_{k}\right)\end{array}\right]$;

In the formula, $\left(\Phi_{i}, \Phi_{j}\right)$ is the inner product of $\Phi_{i}$ and $\Phi_{j}$.

$S_{2}=S_{1}^{T} ; A=\left[a_{k 0}, a_{k 1}, \cdots a_{k n}\right]^{T} ; \lambda=\left[\lambda_{1}, \lambda_{2}\right]^{T} ; T_{1}=\left[g_{k-1}\left(x_{k}\right), g^{\prime}{ }_{k-1}\left(x_{k}\right)\right]^{T} ;$

$T=\left[\left(F, \Phi_{0}\right),\left(F, \Phi_{1}\right), \cdots,\left(F, \Phi_{n}\right)\right]^{T}$, in which: $\Phi_{m}=\left[x_{k}^{m}, x_{k+1}^{m} \cdots x_{k+M-1}^{m}\right]^{T}, F=\left[y_{k}, y_{k+1, \cdots} y_{k+M-1}\right]^{T}$.

From the above matrix equation, we can get the coefficient $a_{k i}(i=1,2,3 \cdots n)$ and $\lambda_{l}(l=1,2)$ in Lagrange function, thus we can get the fitting curve between data points $\left[x_{k}, x_{\mathbf{k}+1}\right]$. In this way, we can finally get the global fitting curve.

\section{Application example}

The following is an example of the application of method Lagrange multiplier in spiral surface fairing problems' measurement. In engineering, when processing and measuring the profile of spiral curved surface's section, micrometer is usually used to measure the major diameter and small diameter of section, However, data accuracy measured by this method is not so ideal. In this article, optical pickup is adopted to continuously measure the profile of spiral surface's section, profile of the whole section is made up of data points, as shown in figure 2.

Partial enlarge the above red box position, as shown in figure 3, we can see because of the influence of such factors as measure device error, workpiece vibration when processing and so on, the measurement of the point cloud data is a closed curve which is not fairing , therefore, the measuring points need to be faired. Because of the measured data is a group of intensive point cloud data (measure 2000 data points of spiral surface outline whose major diameter is about $10 \mathrm{~cm}$ ), thus using existing methods can not effectively fair them.

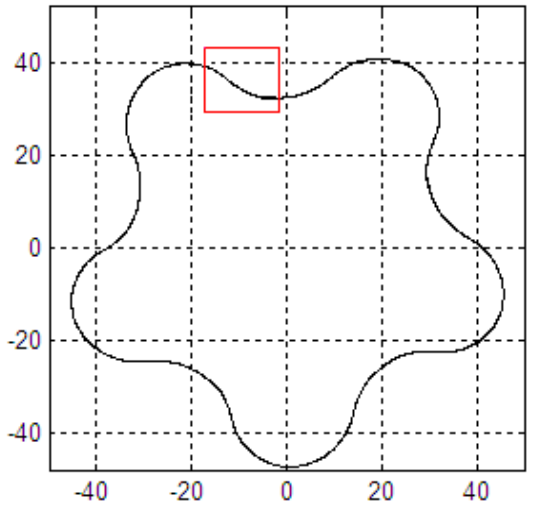

Fig. 2 Section profile

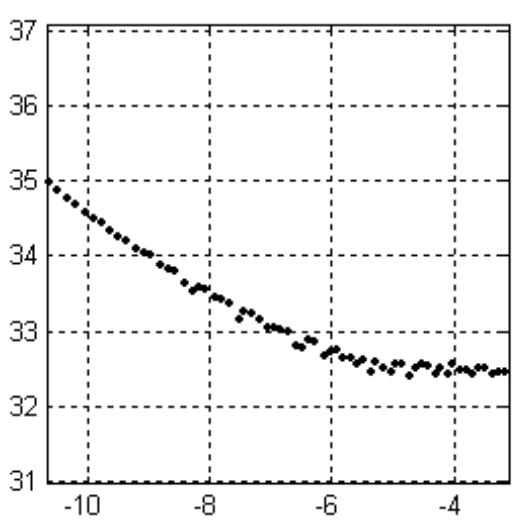

Fig. 3 Partial enlargement contour map of the original spiral surface

As this screw surface profile is a closed curve by made up of data points, so it should be expressed in the form of polar coordinates, namely: $r=r(\theta), \theta \in[0360]$, as shown in figure 4.

Use method Lagrange multiplier proposed in this paper to fairing process point cloud data, select five points as a window, quadratic polynomial as the fitting function, after disposing by programming algorithm on computer, we can get the partial enlargement comparison chart of the graph, as shown in figure 5.Through the comparison of partial enlargement of the curve after fairing and the original data points range, we know that the curve after fairing is continuous, the slight fluctuations appeared on the curve have been faired, and the first derivative of the curve is global continuous, which make it a continuous and smooth curve, furthermore, the faired curve across the center fluctuation range of the original curve's data points, so its fitting precision is higher. 


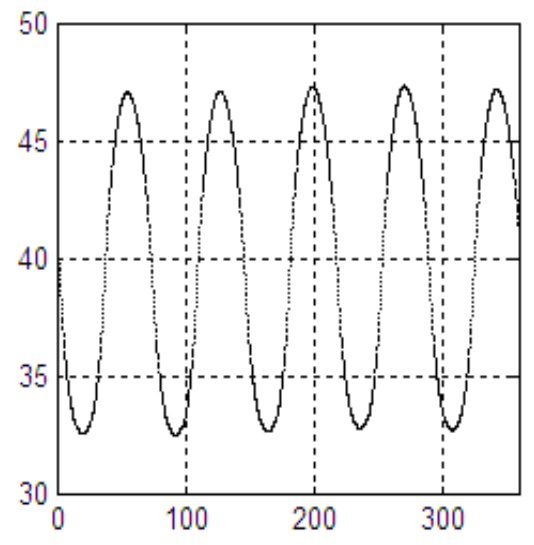

Fig.4 Section profile in polar coordinates

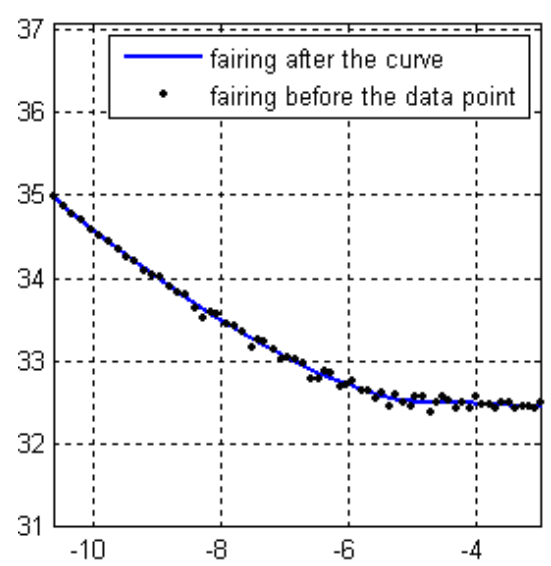

Fig.5 Fairing effect of the curve

\section{Conclusion}

Aiming at the existing problems of the fairing algorithm of point cloud data, this paper put forward a kind of algorithm who is based on Lagrange multiplier to deal with the fairing of point cloud data, modeling and solve the algorithm, finally through the inspection of actual data, proved that the algorithm can not only keep the nature of the curve, more importantly, it realize the curve and it's first derivative's global continuous, proved that the curve fairness effect after disposing is better. At the same time avoid the various abuses produced by using equation of higher degree to fit.

\section{References}

[1] S. Cai, H. Zhang and H.H. Chen. Research of Piecewise Cubic Curve-fitting Method Based on Least-square Principle. Science Technology and Engineering Publishing, 2007(7), p. 352-355.

[2] Y.M. Zhao, G.Z. Zhang and Z.F. Yu. The application study in cloud points disposing with CATIA in Vehicle RE design. Machinery Design \& Manufacture Publishing, 2005(6), p. 80-82.

[3] H. Liu, Y.L. Tao and J.W. Fu. Data processing of scanning beam points-cloud based measuring freeform surface. Modular Machine Tool \& Automatic Manufacturing Technique, 2011(5), p. 77-80. [4] W.X. Li. Partition Fitting Technology in Long-term Trend Modelling Process Concrete Application. World Sci-Tech R \& D Publishing, 2008(3), p. 364-369.

[5] P. Huang. Theory and Methods of Optimization. Tsinghua University Press, Beijing, China, (2009).

[6] Mathematics Department of Tongji University. Advanced Mathematics (the fifth edition). Higher Education Press, Beijing, China, (2006), p. 56-59. 